J. Lake Sci.(湖泊科学), 2017, 29(2): 439-447

DOI 10. 18307/2017. 0220

(C) 2017 by Journal of Lake Sciences

\title{
三峡库区汉丰湖鱼类群落结构的季节变化"
}

\author{
王 敏 ${ }^{1,2}$, 朱峰跃 $^{1}$, 刘绍平 $^{1}, \mathbf{E}^{\text {辛斌 }}{ }^{1}$, 陈大庆 $^{1 * *}$ \\ (1: 中国水产科学研究院长江水产研究所,武汉 430223) \\ (2:华中农业大学水产学院,武汉 430070)
}

\begin{abstract}
摘 要: 为了解汉丰湖鱼类群落结构的季节变化特征, 以便为汉丰湖鱼类资源的合理利用及保护提供理论依据, 于 2014 年 12 月、2015 年 $4 、 7$ 和 10 月按季度共 4 次对汉丰湖鱼类群落结构进行调查与分析. 结果表明:共采集到鱼类 8538 尾,38 种, 隶属于 5 目 9 科 32 属; 其中, 鲤形目鱼类有 28 种, 占总物种数的 $73.68 \%$. 鱼类组成以湖泊定居性种类为主, 瓦氏黄颡 鱼( Pelteobagrus vachelli)、蛇鮈 (Saurogobio dabryi)、银鮈 (Squalidus argentatus)、鲫 (Carassius auratus)、光泽黄颡鱼 (Pelteobagrus nitidus)、鲤(Cyprinus carpio) 和贝氏粲(Hemiculter bleekeri) 为汉丰湖的重要优势种, 占总尾数的 $67.45 \%$. 汉丰湖鱼 类群落 Shannon-Wiener 多样性指数、Pielous 均匀度指数和 Margalef 丰富度指数在冬季最高, 而 Pielous 均匀度指数在不同 季节相对稳定. Jaccard 群落相似性指数较高, 季节间种类相似度较高. 鱼类群落稳定性分析表明, 冬季稳定性最高, 其次 为春季,夏季稳定性最低. 湖泊生境尤其是水位的变化对汉丰湖鱼类群落结构影响明显.
\end{abstract}

关键词: 三峡库区;汉丰湖;鱼类;群落结构;生物多样性;季节变化

\section{Seasonal variations of fish community structure of Lake Hanfeng in Three Gorges Reser- voir region}

\author{
WANG Min ${ }^{1,2}$, ZHU Fengyue ${ }^{1}$, LIU Shaoping ${ }^{1}$, DUAN Xinbin ${ }^{1} \&$ CHEN Daqing ${ }^{1 * *}$ \\ (1: Yangtze River Fisheries Research Institute, Chinese Academy of Fishery Sciences, Wuhan 430223, P.R. China) \\ (2: College of Fisheries, Huazhong Agricultural University, Wuhan 430070, P.R. China)
}

\begin{abstract}
Lake Hanfeng is located in the junction of east and south river in Kaixian county of Chongqing City in middle and upper reaches of Xiaojiang River in the Three Gorges Reservoir region. The change in its water level is dramatic (from $175 \mathrm{~m}$ when the Three Gorges Dam operated to $160 \mathrm{~m}$ in the summer) and mainly dually controlled by Three Gorges Reservoir and water level regulation dam of Lake Hanfeng in Wuyang village. The large fluctuation in water level has great influences on the quality of aquatic organisms including fish within the region but being rarly studied. This study analyzed the seasonal change characteristics of community structure of fish in the lake under the background of changing water level.The survey and analysis of community structure of fish in the Lake Hanfeng was carried out in Dec. 2014 and Apr., Jul., and Oct., 2015.A total of 8538 individuals were taxonomically identified, belonging to 38 species of 9 families in 5 orders. Among those, 28 species belong to Cypriniformes, accounting for $73.68 \%$ of the total, and mainly composed of limnicolous fish.The species number in each season was relatively stable, with 28 , 28, 29, 33 species in spring, summer, autumn and winter, respectively.Pelteobagrus vachelli, Saurogobio dabryi, Squalidus argentatus, Carassius auratus, Pelteobagrus nitidus, Cyprinus carpio and Hemiculter bleekeri were the dominant species, accounting for $67.45 \%$ of the total.Seasonally, the number of dominant species was highest in winter (12), followed by spring (9), summer (11) and autumn (6).The fish diversity index, Shannon-Wiener index, Pielou evenness index and Margalef index was the highest in winter, while the lowest Shannon-Wiener diversity index and Pielous evenness index in summer. There were no significant differences between the seasonal Pielous evenness index and the Shannon-Wiener diversity index. Jaccard index was high, which indicated that more fish species overlapped seasonally. The Jaccard similarity index analysis showed that the similarity index of fish species in different seasons was higher than that in spring and autumn $(83.87 \%)$. Fish community was stablest in winter, followed by
\end{abstract}

* 公益性行业(农业)科研专项经费项目(201303056-5) 资助. 2015-12-14 收稿; 2016-06-16 收修改稿. 王敏 (1991 ), 男, 硕士研究生;E-mail: hznwangmin@163.com.

** 通信作者;E-mail:chdq@yfi.ac.cn. 
spring, autumn and summer. The change in lake habitat, especially fluctuation of water level, had a significant impact on fish community structure in Lake Hanfeng.

Keywords: Three Gorges Reservoir region; Lake Hanfeng; community structure; biodiversity; seasonal variations

鱼类群落是特定水域鱼类种群相互结合的一种结构单元, 鱼类与周围环境及其他物种相互依赖、相互 作用, 组合成具有内在联系与结构特点的整体单元 ${ }^{[1]}$. 水体生境条件的改变会影响鱼类的生存与繁衍, 从而 导致鱼类群落结构及物种多样性发生相应的变化 ${ }^{[2]}$, 这一变化过程不仅可能造成部分功能类群的消失 ${ }^{[3]}$, 进而导致渔业功能的退化, 也会改变生态系统的物流、能流循环途径 ${ }^{[4]}$, 使湖泊生态系统失去自我调控的重 要功能 ${ }^{[5]}$. 因此, 通过鱼类资源调查, 分析生境变化背景下鱼类群落结构的变化特征, 对探讨湖泊生态系统 对环境变化的响应过程具有重要意义.

汉丰湖 $\left(31^{\circ} 10^{\prime} \sim 31^{\circ} 13^{\prime} \mathrm{N}, 108^{\circ} 22^{\prime} \sim 108^{\circ} 28^{\prime} \mathrm{E}\right)$ 位于三峡库区腹地小江上游, 其水位变动受到三峡大坝 和汉丰湖水位调节坝的双重控制. 根据汉丰湖调节坝的计划调度方案, 当三峡水库水位上涨至 $172.8 \mathrm{~m}$ 时, 调节坝闸门开启, 汉丰湖水位与三峡水库同步运行; 水位下降至 $172.8 \mathrm{~m}$ 以下时, 调节坝闸门关闭进行蓄水, 使汉丰湖水位维持在 $172.8 \mathrm{~m}$ 左右 ${ }^{[6]}$, 以此降低因水位大幅度波动对汉丰湖水生态系统的扰动, 但由于截至 目前汉丰湖调节坝还未正式运行, 汉丰湖在不同季节的水位主要受到三峡水库运行的影响, 夏季为低水位 期,冬季为高水位期,水位落差可达 $15 \mathrm{~m}$ 以上.

目前, 关于汉丰湖的研究主要集中在湿地生态恢复潜力 ${ }^{[7]}$ 、水质时空分布 ${ }^{[8]}$ 、浮游植物群落 ${ }^{[9]}$ 等方面. 对于汉丰湖鱼类群落结构特征的研究较少, 仅有丁庆秋等 ${ }^{[10]}$ 在 2012 和 2013 年 2 次对汉丰湖高、低水位下 鱼类资源进行的调查. 针对汉丰湖鱼类群落结构多样性及其季节变化的研究还未见报道. 为此, 作者于 2014 年 12 月以及 2015 年 4、7、10 月对汉丰湖鱼类资源进行了四个季度的调查, 以期进一步了解汉丰湖鱼类群落 结构变化特征,为汉丰湖的渔业管理提供基础参考资料.

\section{1 材料和方法}

\section{1 研究区域概况及样点设置}

重庆汉丰湖位于三峡库区澎溪河中上游的开县境内, 是在开县新城下游 $4.5 \mathrm{~km}$ 处的乌杨村新建水位调 节坝后形成的人工湖, 范围东起乌杨桥水位调节坝, 西至南河大邱坝, 南以新城防护堤高程 $180 \mathrm{~m}$ 为界, 北 到老县城所在的汉丰坝至乌杨坝一线(图 1), 规划常年正常蓄水水位在 $170.28 \mathrm{~m}$ 以上, 水域面积 $14.8 \mathrm{~km}^{2}$, 蓄水量 $8000 \times 10^{4} \mathrm{~m}^{3}$.

汉丰湖由开县城区内东河与南河交汇而成, 依据汉丰湖地理形状及水文条件, 共设置 5 个调查点, 分别 位于湖中心 (HF1、HF2)、东河区域(HF3) 和南河区域(HF4、HF5). 2014 年 12 月及 2015 年 4、7、10 月共 4 次 分别对 5 个点进行鱼类样本采集.

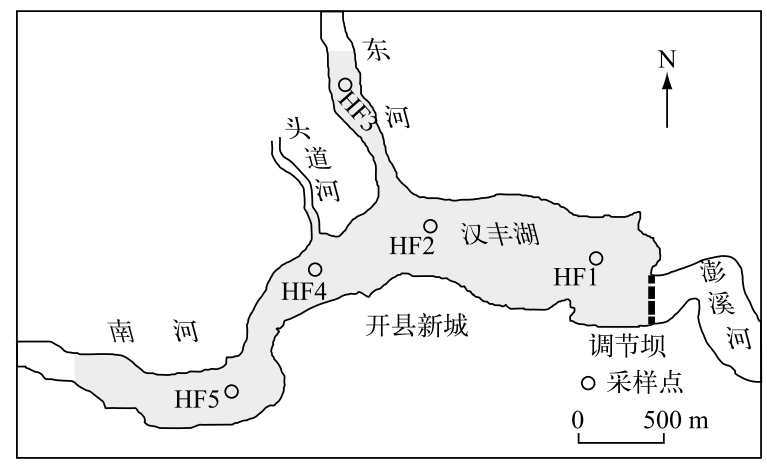

图 1 汉丰湖鱼类调查采样点分布

Fig. 1 The sampling sites of fish resources investigation in Lake Hanfeng 


\section{2 调查方法}

参照《内陆水域渔业自然资源调查手册》 ${ }^{[11]}$ 中的监测方法进行鱼类调查, 采用雇请渔民捕捞的方式开 展调查, 调查的渔具主要为定置刺网, 辅以地笼、犆钩等, 其中定置刺网的网目大小为 $2 \sim 12 \mathrm{~cm}$, 每天下午在 各采样点布设网具, 第 $2 \mathrm{~d}$ 凌晨收取, 每次调查持续捕捞 7 8 d. 同时对市场上售卖的渔获物开展鱼类标本 的补充收集,尽可能对汉丰湖鱼类资源进行全面采集. 依据《中国淡水鱼类检索 $\rangle^{[12]}$ 和《四川鱼类志》 ${ }^{[13]}$ 现 场进行种类鉴定, 并逐尾测定体长 (精确到 $1 \mathrm{~mm}$ ) 及体重 (精确到 $0.1 \mathrm{~g}$ ). 对于少数现场未能鉴定的种类, 采 用 $10 \%$ 的甲醛溶液保存后带回实验室做进一步鉴定和测量.

\section{3 数据分析}

1.3.1 群落优势种 本文采用相对重要性指数 (Index of Relative Importance, IRI) ${ }^{[14]}$ 对汉丰湖鱼类群落优势 种进行度量,其计算公式为:

$$
I R I=(N \%+W \%) F \% \times 10^{4}
$$

式中, $N \%$ 为某物种的数量占总数量的百分比, $W \%$ 为某物种的重量占总重量的百分比, $F \%$ 为此物种在调查 中出现的次数占总调查次数的百分比. 本文将 $I R I>500$ 的物种确定为群落优势种.

1.3.2 鱼类物种相似性指数 采用 Jaccard 群落相似性系数 $\left(J_{\mathrm{S}}\right)^{[15]}$ 衡量不同季节间鱼类物种间的相似性, 其 计算公式为:

$$
J_{\mathrm{S}}=\frac{c}{a+b-c} \times 100 \%
$$

式中, $a$ 为第 1 季节鱼类群落中出现的物种数, $b$ 为第 2 季节鱼类群落中出现的物种数, $c$ 为 2 个季节共有的 物种数.

1.3.3 群落季节更替指数与迁移指数 群落的季节更替指数 (Alternate Index, $A I$ ) 和迁移指数 (Migration Index, $M I)$ 计算公式分别为:

$$
\begin{aligned}
& A I=\frac{C+B}{A-R} \times 100 \\
& M I=\frac{C-B}{A-R} \times 100
\end{aligned}
$$

式中, $A$ 为各季节实际物种数, $C$ 为本季节新迁人物种数, $B$ 为本季节将迁出物种数, $R$ 为 4 次调查中均出现 的物种数. $A I$ 反映物种更替导致群落稳定性降低的节律, 其值越大则表示群落稳定性越小. $M I$ 定义为研究 系统外迁人种与迁出种在群落中的相对比例, 当 $C>B$ 时, $M I$ 为正, 表示迁人种多于迁出种; 当 $C$ 接近于 $B$ 时, $M I$ 为零, 意味着群落趋于动态平衡 ${ }^{[16]}$.

1.3.4 生物多样性 根据 Shannon-Wiener 多样性指数 $\left(H^{\prime}\right) 、$ Margalef 种类丰富度指数 $(D)$ 和 Pielous 均匀度指 数 $\left(J^{\prime}\right)$ 分析汉丰湖鱼类群落多样性 ${ }^{[17-19]}$, 其计算公式分别为:

$$
\begin{gathered}
H^{\prime}=-\sum P_{i} \ln P_{i} \\
D=(S-1) / \ln N \\
J^{\prime}=H^{\prime} / \ln S
\end{gathered}
$$

式中, $S$ 为渔获物中物种的种类数, $N$ 为总渔获物尾数, $P_{i}$ 为第 $i$ 种鱼在总渔获物中所占的个体数比例.

数据的统计分析使用 Excel 2010 和 SPSS 19.0 软件; 采用 Corel DRAW X4 SP2 和 Origin 9.0 绘图.

\section{2 结果}

\section{1 鱼类种类组成和生态类型}

4 次调查在汉丰湖共采集到鱼类样本 8538 尾,经鉴定共有鱼类 38 种,隶属于 5 目 9 科 32 属 (表 1 ). 鲤 科鱼类种类数最多,有 24 种,占总物种数的 $63.16 \%$; 其次是魭科, 5 种, 占 $13.16 \%$; 䱊科 3 种, 占 $7.89 \%$; 胭脂 鱼科、鲇科、鱵科、合鳃鱼科、鮨科和鰕虎鱼科各 1 种, 均占 $2.63 \%$. 调查结果显示, 汉丰湖春、夏、秋、冬 4 个季 节的渔获物种类数除了冬季略高外, 其他季节相对稳定, 春、夏、秋、冬季分别为 $28 、 28 、 29$ 和 33 种. 其中, 汉 
表 1 汉丰湖鱼类种类组成 *

Tab. 1 Species composition of fishes in Lake Hanfeng

\begin{tabular}{|c|c|c|c|c|c|}
\hline 种类 & 生态类型 & 冬季( 12 月) & 春季(4月) & 夏季( 7 月) & 秋季( 10 月) \\
\hline \multicolumn{6}{|l|}{ 胭脂鱼科 Catostomidae } \\
\hline 胭脂鱼 Myxocyprinus asiaticus & $\mathrm{M}, \mathrm{O}$ & + & & & \\
\hline \multicolumn{6}{|l|}{ 鲉科 Cobitidae } \\
\hline 中华沙鳅 Botia superciliaris & $\mathrm{S}, \mathrm{O}$ & + & + & + & + \\
\hline 花斑副沙鳅 Parabotia fasciata & $\mathrm{S}, \mathrm{O}$ & ++ & + & + & ++ \\
\hline 泥鳅 Misgurnus anguillicaudatus & $\mathrm{S}, \mathrm{O}$ & + & + & + & + \\
\hline \multicolumn{6}{|l|}{ 鲤科 Cyprinidae } \\
\hline 马口鱼 Opsariichthys bidens & $\mathrm{S}, \mathrm{P}$ & + & + & + & \\
\hline 草鱼 Ctenopharyngodon idellus & $\mathrm{M}, \mathrm{G}$ & ++ & + & + & + \\
\hline 赤眼鳟 Squaliobarbus curriculus & $\mathrm{M}, \mathrm{O}$ & & & + & \\
\hline 银鲖 Xenocypris argentea & $\mathrm{S}, \mathrm{O}$ & & & + & \\
\hline 似鳊 Pseudobrama simony & $S, G$ & +++ & +++ & +++ & +++ \\
\hline 鲢 Hypophthalmichthys molitrix & $\mathrm{M}, \mathrm{G}$ & ++ & + & + & + \\
\hline 鳙 Aristichthys nobilis & $\mathrm{M}, \mathrm{P}$ & + & + & & \\
\hline 彩石鳑鲏 Rhodeus lighti & $\mathrm{S}, \mathrm{O}$ & + & ++ & & + \\
\hline 高体鳑鲏 Rhodeus ocellatus & $\mathrm{S}, \mathrm{O}$ & + & + & & + \\
\hline 寡鳞飘鱼 Pseudolaubuca engraulis & $\mathrm{S}, \mathrm{O}$ & & & & + \\
\hline 张氏粲 Hemiculter tchangi & $\mathrm{S}, \mathrm{O}$ & +++ & +++ & +++ & ++ \\
\hline 贝氏粲 Hemiculter bleekeri & $\mathrm{S}, \mathrm{O}$ & +++ & +++ & +++ & ++ \\
\hline 尧嘴鲌 Culter ilishaeformis & $\mathrm{S}, \mathrm{P}$ & +++ & +++ & +++ & ++ \\
\hline 蒙古鲌 Culter mongolicus mongolicus & $\mathrm{S}, \mathrm{P}$ & +++ & +++ & +++ & +++ \\
\hline 达氏鲌 Culter dabryi & $\mathrm{S}, \mathrm{P}$ & +++ & +++ & +++ & +++ \\
\hline 唇鳃Hmibarbus labeo & $\mathrm{S}, \mathrm{P}$ & ++ & & & \\
\hline 麦穗鱼 Pseudorasbora parva & $\mathrm{S}, \mathrm{O}$ & & & & ++ \\
\hline 银鮈 Squalidus argentatus & $\mathrm{S}, \mathrm{O}$ & +++ & +++ & +++ & +++ \\
\hline 蛇鮈 Saurogobio dabryi & $\mathrm{S}, \mathrm{O}$ & +++ & +++ & +++ & +++ \\
\hline 吻鮈 Rhinogobio typus & $\mathrm{S}, \mathrm{O}$ & + & & + & \\
\hline 华鲮 Sinilabeo rendahli & $\mathrm{S}, \mathrm{O}$ & + & & + & + \\
\hline 岩原鲤 Procypris rabaudi & $\mathrm{S}, \mathrm{O}$ & + & & & \\
\hline 鲤 Cyprinus carpio & $\mathrm{S}, \mathrm{O}$ & +++ & +++ & ++ & ++ \\
\hline 鲫 Carassius auratus & $\mathrm{S}, \mathrm{O}$ & +++ & +++ & +++ & +++ \\
\hline \multicolumn{6}{|l|}{ 鲇科 Siluridae } \\
\hline 鲇 Silurus asotus & $\mathrm{S}, \mathrm{P}$ & +++ & ++ & ++ & ++ \\
\hline \multicolumn{6}{|l|}{ 鲿科 Bagridae } \\
\hline 黄颡鱼 Pelteobagrus fulvidraco & $\mathrm{S}, \mathrm{P}$ & + & + & ++ & + \\
\hline 瓦氏黄颡鱼 Pelteobagrus vachelli & $\mathrm{S}, \mathrm{P}$ & +++ & +++ & +++ & +++ \\
\hline 光泽黄颡鱼 Pelteobagrus nitidus & $\mathrm{S}, \mathrm{P}$ & +++ & +++ & +++ & +++ \\
\hline 切尾拟鲿 Pseudobagrus truncates & $\mathrm{S}, \mathrm{P}$ & + & & & \\
\hline 大鲑鳠 Mystus macropterus & $\mathrm{S}, \mathrm{P}$ & + & ++ & ++ & ++ \\
\hline \multicolumn{6}{|l|}{ 鱵科 Hemiramphidae } \\
\hline 鮓 Hemiramphus kurumeus & $\mathrm{S}, \mathrm{O}$ & + & + & + & + \\
\hline \multicolumn{6}{|l|}{ 合鳃鱼科 Synbranchidae } \\
\hline 黄鳝 Monopterus albus & $\mathrm{S}, \mathrm{P}$ & & + & + & + \\
\hline \multicolumn{6}{|l|}{ 鮨科 Serranidae } \\
\hline 大眼鳜 Siniperca kneri & $\mathrm{S}, \mathrm{P}$ & + & ++ & +++ & ++ \\
\hline \multicolumn{6}{|l|}{ 鰕虎鱼科 Gobiidae } \\
\hline 子陵栉鰕虎鱼 Ctenogobius giuriuns & $\mathrm{S}, \mathrm{P}$ & ++ & ++ & & + \\
\hline
\end{tabular}

$* \mathrm{~S}$ 为定居性鱼类, $M$ 为洄游性鱼类, $P$ 为肉食性, $G$ 为草食性, $O$ 杂食性, + 表示偶见, ++ 表示出现, +++ 表示出现较多. 
丰湖各季节均有分布的鱼类有 22 种, 占总种数的 $57.89 \%$, 主要为银鮈、瓦氏黄颡鱼、蛇鮈、张氏粲、光泽黄颖 鱼、鲫等. 另外, 胭脂鱼、唇粲和岩原鲤均只在 2014 年 12 月采集到, 而赤眼鳟和银鲖只在 2015 年 7 月采集 到,麦穗鱼只在 2015 年 10 月采集到.

从鱼类生态类型划分 ${ }^{[20]}$ 来看, 汉丰湖水域以湖泊定居性鱼类为主, 种数多达 33 种, 占鱼类物种数的 $86.84 \%$, 为汉丰湖鱼类的主体; 依据食性类型 ${ }^{[11]}$ 来划分, 汉丰湖鱼类可分为肉食性、草食性和杂食性 3 类, 以鲤、鲫和银鮈为典型的杂食性鱼类种类最多,有 20 种,占总物种数的 $52.63 \%$.

\section{2 鱼类优势种组成}

根据相对重要性指数 $(I R I)$ 计算, 汉丰湖鱼类优势种排在前 10 位的鱼类分别为瓦氏黄颡鱼、蛇鮈、银 鮈、鲫、光泽黄滪鱼、鲤、贝氏粲、尧嘴鲌、张氏粲和似鳊 (表 2), 其重量之和占总渔获量的 $72.07 \%$, 尾数之和 占总尾数的 $80.43 \%$. 其中瓦氏黄颖鱼作为汉丰湖最优势种, 占总尾数的 $16.28 \%$, 总渔获量的 $18.18 \%$, 在春、 夏和秋季均处于第一优势种. 优势种中鲤个体较大, 平均体重达 $243.4 \mathrm{~g}$, 其他种类均为中、小型鱼类或幼鱼. 渔获物中绝大多数鱼类体长不超过 $150 \mathrm{~mm}$, 质量小于 $30 \mathrm{~g}$ 的小型鱼类占 $66.94 \%$.

表 2 汉丰湖主要鱼类体长和体重分布

Tab.2 Distribution of body length and weight of main fishes in Lake Hanfeng

\begin{tabular}{|c|c|c|c|c|c|}
\hline 优势种 & $I R I$ & 体长范围/mm & 平均体长 $/ \mathrm{mm}$ & 体重范围/g & 平均体重/g \\
\hline 瓦氏黄滪鱼 & 3446 & $66 \sim 235$ & 116 & $4.0 \sim 230.0$ & 28.2 \\
\hline 蛇鮈 & 2037 & $50 \sim 148$ & 98 & $1.7 \sim 35.9$ & 11.0 \\
\hline 银鮈 & 1842 & $45 \sim 116$ & 74 & $1.1 \sim 22.9$ & 6.1 \\
\hline 鲫 & 1257 & $44 \sim 358$ & 119 & $2.1 \sim 829.6$ & 58.3 \\
\hline 光泽黄颡鱼 & 1095 & $85 \sim 138$ & 105 & $6.3 \sim 46.8$ & 15.1 \\
\hline 鲤 & 869 & $64 \sim 365$ & 181 & $5.7 \sim 1150.0$ & 243.4 \\
\hline 贝氏粲 & 778 & $60 \sim 210$ & 125 & $2.4 \sim 117.7$ & 33.0 \\
\hline 翅嘴鲌 & 751 & $16 \sim 310$ & 188 & $7.4 \sim 224.0$ & 74.1 \\
\hline 张氏粲 & 750 & $64 \sim 190$ & 101 & $2.8 \sim 95.8$ & 13.9 \\
\hline 似鯿 & 688 & $69 \sim 165$ & 107 & $3.3 \sim 71.2$ & 23.7 \\
\hline
\end{tabular}

优势种在各季节均占有一定优势, 但不同季节优势种组成也存在部分波动 (表 3). 在季节分布方面, 瓦 氏黄滪鱼和蛇鮈在春、秋季优势度明显, 而蛇鮈在夏季低水位时期优势度不明显, 鲫在冬季所占比例较高, 张氏粲在夏、冬季均具有一定的优势. 从季节变化来看, 优势种数量大体呈下降趋势, 其中冬季优势种数量 最多 (12 种), 其次为春季 ( 9 种 $)$,夏季和秋季分别为 11 和 6 种.

表 3 汉丰湖不同季节鱼类优势种组成

Tab.3 Composition of dominate fish species among various seasons in Lake Hanfeng

\begin{tabular}{|c|c|c|c|c|c|c|c|c|}
\hline \multirow{2}{*}{ 优势种 } & \multicolumn{2}{|c|}{2014 年 12 月 } & \multicolumn{2}{|c|}{2015 年 4 月 } & \multicolumn{2}{|c|}{2015 年 7 月 } & \multicolumn{2}{|c|}{2015 年 10 月 } \\
\hline & $N \%$ & IRI & $N \%$ & IRI & $\mathrm{N} \%$ & $I R I$ & $N \%$ & IRI \\
\hline 瓦氏黄颡鱼 & 9.84 & 1967 & 16.58 & 3150 & 15.78 & 3079 & 19.99 & 5201 \\
\hline 蛇鮈 & 11.90 & 2195 & 24.21 & 3004 & 1.44 & 121 & 22.03 & 3320 \\
\hline 银鮈 & 8.77 & 1255 & 13.40 & 1698 & 30.21 & 3037 & 9.95 & 1076 \\
\hline 鲫 & 9.21 & 2207 & 10.22 & 2596 & 1.23 & 312 & 2.24 & 524 \\
\hline 光泽黄颡鱼 & 2.59 & 472 & 4.75 & 834 & 5.90 & 885 & 12.18 & 2095 \\
\hline 鲤 & 6.53 & 1733 & 2.65 & 1558 & 0.34 & 645 & 0.25 & 206 \\
\hline 贝氏粲 & 5.19 & 932 & 5.18 & 1214 & 6.11 & 880 & 1.34 & 158 \\
\hline 翅嘴鲌 & 3.31 & 728 & 1.97 & 650 & 3.99 & 1872 & 0.55 & 88 \\
\hline 张氏粲 & 12.08 & 2005 & 1.08 & 174 & 15.95 & 2241 & 1.04 & 81 \\
\hline 似鳊 & 8.14 & 1557 & 3.08 & 469 & 5.30 & 1183 & 1.54 & 180 \\
\hline
\end{tabular}


表 4 汉丰湖不同季节鱼类相似性指数 *

Tab.4 The Jaccard similarity index of fishes among various seasons in Lake Hanfeng

\begin{tabular}{ccccc}
\hline 季节 & $\begin{array}{c}\text { 冬季 } \\
\text { 12月 }\end{array}$ & $\begin{array}{c}\text { 春季 } \\
4 \text { 月 }\end{array}$ & $\begin{array}{c}\text { 夏季 } \\
7 \text { 月 }\end{array}$ & $\begin{array}{c}\text { 秋季 } \\
10 \text { 月 }\end{array}$ \\
\hline 冬季 & & 27 & 25 & 26 \\
春季 & $79.41 \%$ & & 24 & 26 \\
夏季 & $69.44 \%$ & $75.00 \%$ & & 24 \\
秋季 & $72.22 \%$ & $83.87 \%$ & $72.73 \%$ & \\
\hline
\end{tabular}

* 对角线上方为共有种数, 下方为 Jaccard 相似性指数.

鱼类相似性指数最低( 表 4).

\section{4 群落多样性的季节变化}

汉丰湖鱼类 $H^{\prime}$ 值变动范围为 $2.21 \sim 2.56, D$ 值为 $3.35 \sim 4.12, J^{\prime}$ 值为 $0.67 \sim 0.73 . H^{\prime} 、 D$ 和 $J^{\prime}$ 值均在冬季最 高, 其他 3 个季节较接近 (图 3 ). $J^{\prime}$ 和 $H^{\prime}$ 值相对稳定, 通过单因素方差分析 (ANOVA), 季节间 $J^{\prime}$ 和 $H^{\prime}$ 值差异 均不显著 $(P>0.05)$.

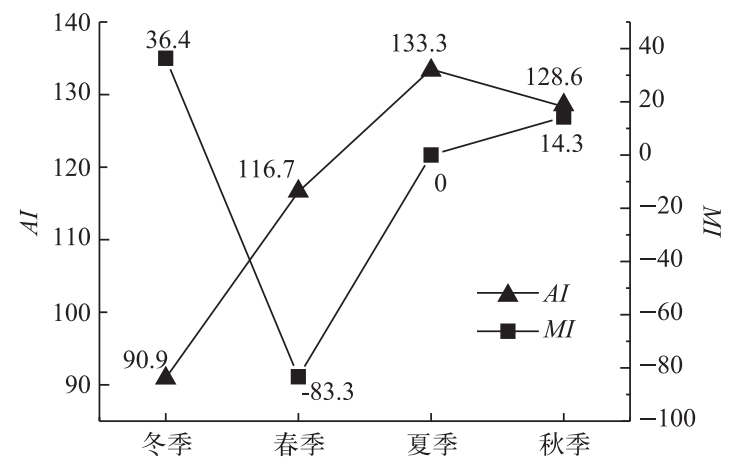

图 2 汉丰湖鱼类群落更替指数和迁移指数的季节变化

Fig.2 Seasonal changes of replacement index and Migration Index of fish community in Lake Hanfeng

\section{3 鱼类群落的季节更替及季节间相似性}

汉丰湖夏、秋季的季节更替指数 $(A I)$ 比冬、春季 高, 表明夏、秋季的群落稳定性比冬、春季低, 冬季稳 定性最高. 从迁移指数 $(M I)$ 的季节变化可见, 秋、冬 季 $M I$ 值为正值, 表明秋、冬季迁人种均多于迁出种; 夏季 $M I$ 值为 0 , 说明鱼类群落趋于动态平衡; 春季 $M I$ 值为负值, 表明春季迁出种明显大于迁人种. 鱼 类迁人量最大的季节为冬季, 其次为秋季, 而迁出量 最大的季节为春季( 图 2).

汉丰湖相邻季节 Jaccard 相似性指数变化范围为 $69.44 \% \sim 83.87 \%$, 表明汉丰湖不同季节鱼类物种相 似性较高, 其中春、秋季相似性指数最大, 而夏、冬季

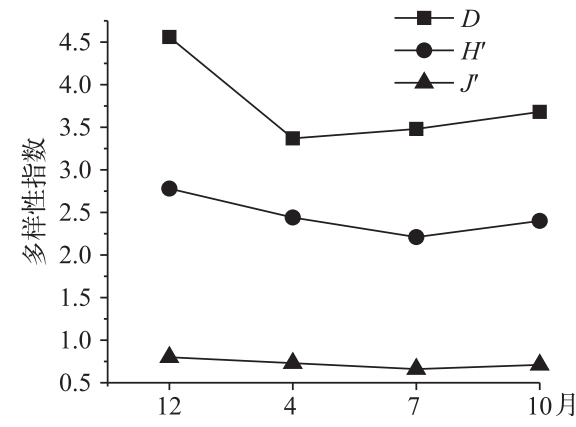

图 3 汉丰湖鱼类生物多样性指数季节变化

Fig. 3 Seasonal variations of fish species biodiversity indices in Lake Hanfeng

\section{3 讨论}

\section{1 调查网具的选择}

调查网具的选择性在一定程度上会影响鱼类的多样性指数及丰富度指数,单一的网具很难捕获到调查 区域的所有鱼类. 在鱼类群落的调查采样中, 针对研究水域环境状况和对象所采用的网具会有所不同, 长江 中上游主要作业网具有定置刺网、流刺网、地笼和扳罾等. 流刺网主要用于捕捞中上层鱼类(如刀鲚、粲等), 无法捕捞底层鱼类 ${ }^{[21]}$. 地笼是一种常用捕捞网具, 对捕捞底层鱼类如黄颡鱼和鲇效果明显. 扳罾是一种定 置性网具, 在水中不能移动, 捕获底层鱼类几率较小. 定置刺网的优点是可通过调节捕捞到各个水层的鱼 类, 采样方便、易行且成本较低, 因此本研究主要选用定置刺网调查汉丰湖的鱼类群落结构. 考虑到单一调 查渔具的局限性, 本次采样作者也搜集了渔民通过使用地笼、饵钩等多种形式捕捞的渔获物进行调查分析, 以期更好地反映汉丰湖鱼类群落结构及物种多样性的动态变化.

\section{2 汉丰湖鱼类群落组成变化}

根据本研究的调查, 汉丰湖鱼类群落显示出以湖泊定居性和小型鱼类为主的特征, 这一结果与以往对 


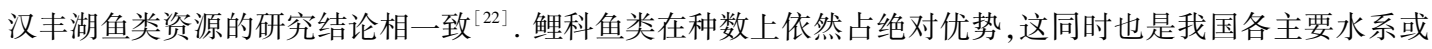
水体鱼类区系组成的共同特点 ${ }^{[23]}$. 本研究在汉丰湖水域共捕获鱼类 38 种, 与 2012-2013 年调查结果 ${ }^{[10]}$ 相 比,鱼类组成发生了一定变化. 调查中发现了胭脂鱼和岩原鲤, 这表明地方实施的保护性增殖放流措施开始 起到一定的作用; 而长薄䱊、铜鱼、圆口铜鱼等喜流水性重要经济鱼类在 4 次采样中均未发现; 与 2011 年李 斌等 ${ }^{[22]}$ 调查相比, 瓦氏黄颖鱼、光泽黄豞鱼、蛇鮈等底层鱼类在渔获物中所占比例上升趋势明显, 已成为渔 民的主要捕捞对象, 而鲇、宽鯺鱲等传统主要捕捞对象在渔获物中所占比例则显著降低. 这表明汉丰湖调节 坝建成后, 水的流速和水体状况及生态环境的变化不利于喜激流性鱼类的生存, 更有利于一些喜静水 (或缓 流水) 的杂食性底层鱼类的生存 ${ }^{[24]}$.

自 2008 年开始汉丰湖连续开展了多次大规模增殖放流活动, 放流品种主要包括草鱼、鲢、鳙、胭脂鱼、 岩原鲤等经济鱼类. 从渔获物中的鱼类组成来看, 这些放流鱼种在渔获物中占比并不高, 草鱼在 4 次采样中 所占的重量比分别为 $9.05 \% 、 8.12 \% 、 3.11 \%$ 和 $2.86 \%$, 鲢在 4 次采样中总共捕获到 7 尾, 而鳙则只在 2014 年 12 月和 2015 年 4 月捕获到 5 尾, 后 2 次调查中再未发现. 造成这一局面的原因除了与汉丰湖捕捞压力较大 和水域生态环境的变动 ${ }^{[25]}$ 有关外, 汉丰湖平缓的水体条件无法满足鲢、鳙等鱼类正常的自然繁殖, 自然资源 补充严重不足也是重要的影响因素.

\section{3 鱼类群落多样性变化}

鱼类多样性指数能够反映群落结构稳定性, 从种群和种群个体数的均匀性两方面衡量群落结构 ${ }^{[26]}$. 群 落物种越丰富, 各种类个体数分布越均匀, 多样性指数越高, 均匀性指数越高 ${ }^{[27]}$. 本研究显示, 汉丰湖鱼类 群落 Shannon-Wiener 多样性指数和 Pielous 均匀度指数呈现显著的季节性变化, 表现为冬季 $>$ 春季 $>$ 秋季 $>$ 夏 季, 而 Margalef 丰富度指数则表现为冬季>秋季>夏季>春季. 而从群落季节更替指数变化来看, 夏、秋季更替 指数较冬、春季高, 说明夏、秋季鱼类群落结构稳定性较低. 这主要是由于汉丰湖夏季处于低水位运行, 致使 栖息环境锐减, 水生植被破坏, 加之环湖库岸开发建设及过度捕捞等因素造成了鱼类结构的变化, 影响了鱼 类群落多样性和稳定性; 而其他季节水位相对较高, 水生植被资源丰富, 随着外来营养物质的汇人, 饵料会 得到不断补充, 这都极大改善了鱼类生境; 因此, 不同季节鱼类群落多样性的差别一定程度上能反映出鱼类 群落结构与湖泊环境因子相适应的特征.

\section{4 汉丰湖鱼类面临的主要问题及保护措施}

汉丰湖调节坝建成后, 汉丰湖作为三峡水库的“库中库”存在. 通过调节坝的调蓄作用,在一定程度上降 低了三峡水库水位变化给汉丰湖生境带来的剧烈扰动, 但鉴于目前调节坝并未正式运行, 汉丰湖内部水位 在季节间仍然存在较大幅度的波动, 不利于水体生态系统向稳态发展. 调查发现, 目前汉丰湖对捕捞强度的 控制力度仍然不足, 非法电捕鱼的现象仍时有发生, 这显然不利于保护汉丰湖鱼类多样性和防止鱼类低龄 化、小型化; 同时调查也发现, 在高水位时期汉丰湖部分区域发生了藻类水华现象, 此时已进入秋冬季节, 水 温开始下降, 故水华的出现可能与蓄水引发水流减缓、水体滞留时间较长有关, 尽管涉及范围不是很大, 还 未大规模爆发,但已表明汉丰湖水体存在一定富营养化现象.

因此,建议采取如下保护措施: (1) 加强渔业行政执法管理. 渔政部门进一步完善禁渔期制度,如合理延 长禁渔时间及范围; 大力宣传保护鱼类资源的意义, 严禁渔民私自驾驶船舶进人汉丰湖保护区内从事违法 捕捞; 在汉丰湖区域内设置警示标语, 公布举报电话, 实施常年昼夜巡查, 坚决禁止电鱼、毒鱼等危害鱼类资 源的严重违法行为, 以此加强对汉丰湖鱼类资源保护力度; (2) 建立人工增殖放流长效机制. 鱼类资源仅仅 依靠自然增殖是非常缓慢的, 汉丰湖应发展以鱼类增殖放流为主的天然生态渔业模式, 有计划地开展人工 增殖放流, 汉丰湖放流品种应以鲢、鳙滤食性鱼类为主, 适当放流草鱼、胭脂鱼、岩原鲤等品种. 通过增殖放 流在补充库区水域经济鱼类和珍稀鱼类种群数量, 保护库区生物多样性的同时, 部分鱼类还可以滤食水中 的藻类和其他浮游生物, 能有效净化水质, 改善库区渔业水域生态环境, 实现汉丰湖渔业的可持续发展.

\section{4 参考文献}

[ 1 ] Ye Fuliang ed. Fish ecology. Guangzhou: Guangdong Higher Education Press, 2002: 179-195. [ 叶富良. 鱼类生态学. 广州: 广东高等教育出版社, 2002: 179-195.] 
[ 2 ] Sutela T, Vehanen T. Effects of water-level regulation on the nearshore fish community in boreal lakes. Hydrobiologia, 2009, 613(1): 13-20.

[ 3 ] Zhang Yi, Hu Ren, Xiao Lijuan et al. Comparative analysis of succession of the phytoplankton functional groupsin two reservoirs with different hydrodynamics in Southern China. Ecology and Environmental Sciences, 2012, 21(1) : 107-117. [张 怡, 胡韧, 肖利娟等. 南亚热带座不同水文动态的水库浮游植物的功能类群演替比较. 生态环境学报, 2012,21 (1) : 107-117.]

[ 4 ] Frauendorf TC, Colón-Gaud C, Whiles MR et al. Energy flow and the trophic basis of macroinvertebrate and amphibian production in a neotropical stream food web. Freshwater Biology, 2013, 91(7) : 28-35.

[ 5 ] Scheffer M, Carpenter S, Foleyj A et al. Catastrophic shifts in ecosystems. Nature, 2001, 413: 591-596.

[6] Li Bo, Yuan Xingzhong, Xiong Sen et al. Preliminary study on the landscape dike-pond system in the drawdown zone of urban area: A case study on the Lake Hanfeng in Kaixian of Chongqing. Journal of Chongqing Normal University: Natural Science, 2013, 31(6) : 51-54. DOI: 10.11721/cqnuj20130610. [李波, 袁兴中, 熊森等. 城市消落带景观基塘系统 设计初探——以重庆开县汉丰湖为例. 重庆师范大学学报: 自然科学版, 2013, 31(6): 51-54.]

[ 7 ] Martin JH, Li Bo, Wang Qiang et al. Potential for wetland restoration in the drawdown zone of Lake Hanfeng. Journal of Chongqing Normal University: Natural Science, 2012, 29(3): 4-7. [Martin JH, 李波, 王强等. 重庆开县汉丰湖湿地 生态恢复的潜力. 重庆师范大学学报: 自然科学版, 2012, 29(3) : 4-7.]

[ 8 ] Huang Qi, He Binghui, Zhao Xiulan et al. Analysis on spatiotemporal variation characteristics of water quality of Lake Hanfeng in Three Gorges Reservoir Region of China. Journal of Southwest University: Natural Science, 2016, 38(3) : 136142. [ 黄祺, 何丙辉, 赵秀兰等. 三峡库区汉丰湖水质的时空变化特征分析. 西南大学学报: 自然科学版, 2016, 38 (3) : 136-142.]

[ 9 ] Wang Yufei, Zhao Xiulan, He Binghui et al. Canonical correspondence analysis of summer phytoplankton community and its environmental factors in Lake Hanfeng. Environmental Science, 2015, 36(3) : 922-927. DOI: 10.13227/j.hjkx.2015. 03.022. [王宇飞, 赵秀兰, 何丙辉等. 汉丰湖夏季浮游植物群落与环境因子的典范对应分析. 环境科学, 2015,36 (3) : 922-927.]

[10] Ding Qingqiu, Peng Jianhua, Yang Zhi et al. Variation in the Lake Hanfeng fishery resource between low and high water levels in Three Gorges Reservoir. Journal of Hydroecology, 2015, 36(3) : 1-9. DOI : 10.15928/j. 1674-3075. 2015.03. 001. [丁庆秋, 彭建华, 杨志等. 三峡水库高、低水位下汉丰湖鱼类资源变化特征. 水生态学杂志, 2015, 36(3) : 1-9. ]

[11] Zhang Jueming, He Zhihui eds. Investigation handbook of fisheries natural resources in inland waters. Beijing: Agriculture Press, 1991：1-461. [张觉明, 何志辉. 内陆水域鱼类资源调查手册. 北京: 农业出版社, 1991: 1-461.]

[12] Zhu Songquan ed. Synopsis of freshwater fishes of China. Nanjing: Jiangsu Publishing House of Science and Technology, 1995: 1-549. [ 朱松泉. 中国淡水鱼类检索. 南京: 江苏科学技术出版社, 1995: 1-549.]

[13] Ding Ruihua ed. The fish of Sichuan. Chengdu: Sichuan Publishing House of Science and Technology, 1994: 117-522. [丁瑞华. 四川鱼类志. 成都: 四川科学技术出版社, 1994: 117-522. ]

[14] Pinkas L, Oliphant MS, Iverson K. Food habits of albacore, bluefin tuna, and bonito in California waters. Fish Bulletin, 1971, 152 : 1-10.

[15] Jaccard P. The distribution of flora in the alpine zone. New Phytologist, 1912, 11: 37-50.

[16] Zhu Xinhua, Wu Hezhou, Xu Fengshan et al. A study on seasonal changes of the fish communities in the Yellow Sea and the Bohai Sea. Acta Oceanologica Sinica, 1994, 16(3): 102-112. [ 朱金华, 吴鹤洲, 徐凤山等. 黄渤海沿岸水域游泳 动物群落多样性及其相关因素的研究. 海洋学报, 1994, 16(3) : 102-112.]

[17] Shannon CE, Wiener WJ. The mathematical theory of communication. Urbana: University of Illinois Press, 1949,19 (7): 1 .

[18] Margalef DR. Information theory in ecology. International Journal of General Systems, 1958, 3(1) : 36-71.

[19] Pielou EC. Ecological diversity. New York: John Wiley and Sons, 1975.

[20] Chen Yiyu ed. Fauna Sinica, Osteichthyes, Cypriniformes II. Beijing: Science Press, 1998: 19-444. [ 陈宜瑜. 中国动物 志・硬骨鱼纲・鲤形目 (中卷). 北京: 科学出版社, 1998: 19-444.]

[21] Shi Weigang, Liu Kai, Zhang Minying et al. Changes of biodiversity of fishery species in the lower reaches of the Yangtze River during the spring closed season. J Lake Sci, 2005, 17(2) : 169-175. DOI: 10.18307/2005.0213. [施炜纲, 刘凯, 
张敏芗等. 春季禁渔期间长江下游鱼虾蟹类物种多样性变动(2001-2004 年). 湖泊科学, 2005, 17(2) : 169-175.]

[22] Li Bin, Jiang Xing, Wang Zhijian et al. The current situation of fishery resources in the Xiaojiang River of the Three Gorges Reservoir. Freshwater Fisheries, 2011, 41(6) : 37-42. [李斌, 江星, 王志坚等. 三峡库区蓄水后小江鱼类资源现 状. 淡水渔业, 2011, 41(6): 37-42.]

[23] Chen YF, Chen YY, He DK. Biodiversity in the Yangtze River fauna and distribution of fishes. Lehthyol, 2002, 42: 161-171.

[24] Shao Xiangyang, Li Daofeng, Cai Qinghua. The composition of the fish community in Xiangxi Bay and resources evaluation. Acta Hydrobiologica Sinca, 2006, 30(1) : 70-74. [ 邵向阳, 黎道丰, 蔡庆华. 香溪河鱼类群落组成及资源评价. 水生生物学报, 2006, 30(1): 70-74.]

[25] Yang Feng, Yao Weizhi, Deng Huatang et al. The current situation of fish resources in the Daning River after the impoundment of the Three Gorges Reservoir. Freshwater Fisheries, 2013, 43(4): 51-57. [杨峰, 姚维志, 邓华堂等. 三峡库区蓄 水后大宁河鱼类资源现状研究. 淡水渔业, 2013, 43(4) : 51-57.]

[26] Li Lingzhi, Wang Lei, Huang Hongliang et al. Study on the component of gill-net catches around the south region of the Yellow Sea in summer. Marine Sciences, 2009, 33(3): 36-40. [李灵智, 王否, 黄洪亮等. 夏季黄海南部沿海表层流 刺网渔获物组成分析. 海洋科学, 2009, 33(3): 36-40.]

[27] Matthews WJ ed. Patterns in freshwater fish ecology. New York: Chapman and Hall Publishers, 1998. 